\title{
A Rare Cause of Sciatica: Sciatic Nerve Schwannoma - A Case Report
}

\author{
Hyun-Jun Park, Myung-Hoon Shin, Jong-Tae Kim, Du-Yong Choi \\ Departments of Neurosurgery, Incheon St Mary's Hospital, College of Medicine, The Catholic University, Incheon, Republic of Korea
}

Corresponding author: Myung-Hoon Shin, MD, PhD Department of Neurosurgery, Incheon St Mary's

Hospital, College of Medicine, The Catholic University of Korea, 56 Dongsu-ro, Bupyeong-gu, Incheon

403-720, Republic of Korea

Tel: +82-32-280-5973

Fax: +82-32-280-5991

E-mail: novice97@naver.com

Received: October 29, 2020

Revised: December 10, 2020

Accepted: December 23, 2020
Herein we report a rare case of a sciatic nerve schwannoma causing in a 63-year-old female. A sciatic localization is rare accounting for $<1 \%$ of all schwannomas. They often lead to misdiagnosis or delayed diagnosis owing to confusion with other common causes of sciatica, such as lumbar herniated disc or spinal stenosis. Magnetic Resonance Image (MRI) is a gold diagnostic tool, and the final diagnosis is made by the histological examination. A standard surgical technique with guidance by neurophysiologic monitoring allows in most of the cases an uncomplicated removal of the tumor. Although being a rare occurrence, a thorough clinical and radiological evaluation of the sciatic nerve should be taken into consideration whenever sciatica is not otherwise explained.

Key Words: Schwannoma, Sciatic nerve, Sciatica

\section{INTRODUCTION}

Schwannomas are the most common peripheral nerve sheath tumors8), originating from well-differentiated Schwann cells of the neural sheath of motor and sensory nerves ${ }^{21}$. They are often solitary, circumscribed and encapsulated by epineurium and grow eccentrically, displacing the nerve root ${ }^{8,22}$. A malignant potential has been reported in less than $5 \%$ of cases and the reported incidence of benign schwannoma is estimated to occur in six patients per $100,000^{24}$. Sporadic schwannomas have no predilection toward sex or race, and offen affect patients aged 20-50 years ${ }^{20}$. They may occur anywhere in all cranial and peripheral nerves except the optic and olfactory nerves.

Schwannomas of the sciatic nerve, which is the largest nerve of the human body, are uncommon (with a frequency of $<1 \%$ of all schwannomas) ${ }^{20}$. They could often raise confusion with other known causes of sciatica, such as lumbar degenerative diseases and its diagnosis might be challenging, leading to a late correct diagnosis in many cases ${ }^{24}$.

Here, we present the case of sciatic nerve schwannoma in a middleaged female subject who presented with the walking difficulty associated with persistent sciatica.

\section{CASE REPORT}

A 63-year-old female presented with pain in the right leg VAS $8 / 10$ ), which presented for the past 5 years and progressively wor- sened over time. The pain started in the right buttock area and it radiated down to the lateral thigh, the back of the leg and the dorsum of the foot. The symptom was also present at rest and woke the patient up from sleep during the night. A lumbosacral Magnetic Resonance Image (MRI) scan demonstrated postoperative laminectomy at L4-5 level (Figure 1A, 1B), which she already has been performed under the diagnosis of spinal stenosis 4 years ago. However, lumbar decompressive surgery failed to relieve her symptom and during the intervening time before the admission, the patient underwent several times of selective nerve root blocks. Following the nerve root block, the relieving effect lasted only for several hours, less than a day at best.

The patient had a history of pulmonary thromboembolism and had been medicated antiplatelet agent for a year. It gave rise to a suspicion of peripheral arterial disease of the legs however, both
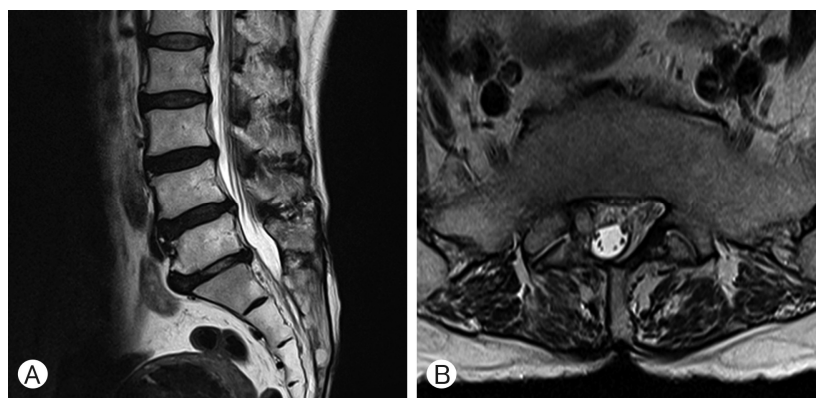

Figure 1. (A): T2 sagittal MRI showing disc space narrowing and protruded disc at the level of L4-5 and L5-S1. (B): T2 axial MRI showing right partial hemilaminectomy of $L 5$. 
ultrasound scanning and Computed Tomography (CT) angiogram showed negative results. Besides, electromyography and nerve conduction test documented negative findings and were not contributory. Multiple opinions were sought. The sciatica was thought to originate from compression by piriformis muscle and the patient was sent to the pain clinic to get piriformis block. Ultrasound scanning was performed to localize the piriformis muscle and it revealed a hypoechoic mass in the gluteal region. Subsequent MRI of the gluteal and thigh region was then performed, and it finally found a rounded and heterogeneously enhanced tumor $(2.5 \times 2 \times 3.7 \mathrm{~cm}$ diameter, Figure 2). It went along the course of the right sciatic nerve, posteriorly to the greater trochanter of the right femur. The physical and neurological examination revealed significant pain related to compression of the posterior proximal region of the thigh although the mass was hardly palpable. There was no remarkable motor deficit but hyporeflexia of the right ankle reflex was noted.

The patient was operated on with microsurgical enucleation with neurophysiological monitoring. The patient was positioned in a left lateral decubitus position and a linear skin incision was made over the greater trochanter. Following the dissection of the iliotibial band and tensor fascia latae, a well-circumscribed, firm, the encapsulated tumor was found originating from the sciatic nerve sheath (Figure 3A). The nerve sheath was dissected, and the tumor was enucleated from the nerve fascicles, which were incorporated within the capsule. Complete tumor resection was achieved with the preservation of the functioning fascicles (Figure 3B). Histological analysis of the
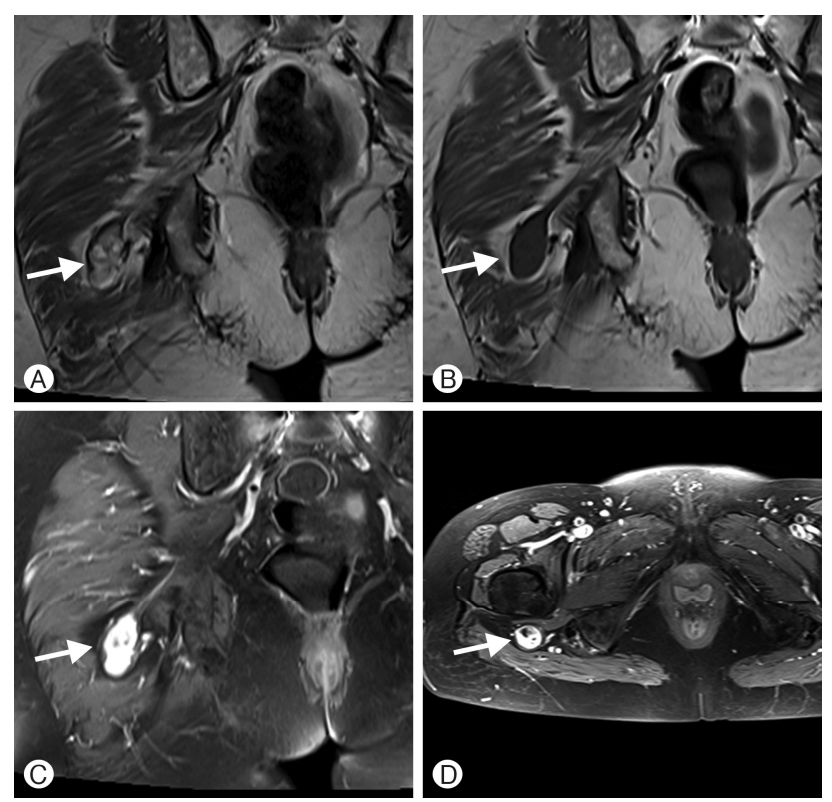

Figure 2. Pelvic MRI showing the sciatic nerve schwannoma (white arrow). (A): T2 coronal view of the pelvic MRI showing an oval, well circumscribed lesion lying posterior to the right greater tronchanter. (B): The mass was homogenously low signal intensity on T2 coronal view. (C), (D): The mass shows a peripheral hyperintensity and a homogenous hypointense center following gandolinium enhancement known as a "target sign". tumor confirmed the diagnosis of a sciatic nerve schwannoma, owing to the presence of high and low cellular regions called Antoni $A$ and B areas, respectively (Figure 4).

The postoperative course was uneventful. Prompt and significant decrease of pain (VAS 1/10) and paresthesia were noted allowing rapid mobilization. There was no complication or any newly developed neurological deficits.

\section{DISCUSSION}

Sciatica is generally referred to pain radiating the leg, usually below the knee and into the foot and toes. It tends to approximate the dermatomal distribution of the affected nerve root, mostly L5, $\mathrm{S} 1$ and often presents paresthesia and numbness in a similar distribution. It may accompany by neurologic deficits such as muscle weakness and reflex change. As with lower back pain is terminology for a symptom rather than a specific diagnosis and typical pathologies included lumbar disc hemiation, lumbar canal, or foraminal stenosis. Generally, the main cause of the pain is believed to be attributable to the inflammatory changes resulting from irritation or compression of the affected nerve root by its surrounding structures ${ }^{12}$. Another
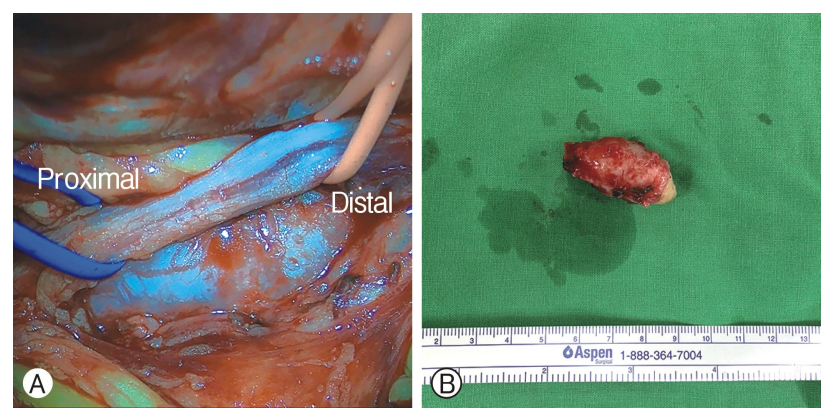

Figure 3. (A): Sciatic nerve and tumor exposed through a transgluteal approach. (B): Gross image of the excised tumor.

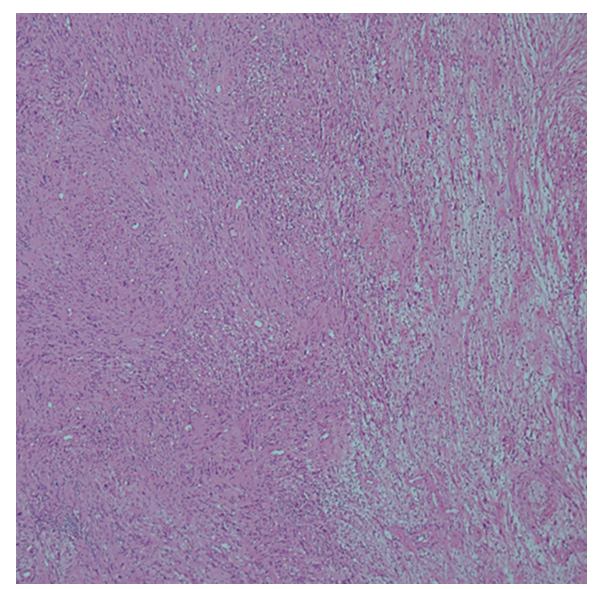

Figure 4. Histopathology of spindle cells showing hyper-celluar (Antoni A) and hypocellular (Antoni B) areas (Hematoxylin-Eosin stain/magnification, $\times 40$ ). 
potential diagnosis of sciatica includes spinal tumors, spinal epidural abscess/hematoma, myositis ossificans of biceps muscle, facet syndrome, lumbar instability, sacroiliitis, sciatic neuritis, and peripheral nerve sheath tumors ${ }^{10}$.

The neoplastic involvement of the sciatic nerve has occurred in soff tissue malignancies, sarcoidosis, lymphoma, infiltrating intermuscular lipomas and fibrolipomatous hamartoma ${ }^{6}$. Filler et al. ${ }^{7}$ investigated the origin of non-discogenic sciatica in 239 patients and reported that sciatic nerve tumors represented only $1.7 \%$ of all cases. Moreover, the incidence of sciatic nerve schwannoma is quite rare that six cases per million were reported to occur ${ }^{19,25}$. Given the fact that schwannomas have a long subclinical course and pain from sciatic nerve schwannoma may simulated sciatica caused by a lumbar disc herniation or spinal stenosis, their clinical presentation is misleading and could lead to late diagnosis (Table 1). The patient in the current case had suffered from sciatic pain for 5 years and was performed lumbar decompressive surgery under the diagnosis of spinal stenosis however, sciatica persisted until the removal of the sciatic schwannoma.

A clinical history of a long-lasting radicular pain being unresponsive to pain medication and rest should raise a suspicion of non-discogenic sciatica and a peripheral nerve lesion. Especially, patients with a negative straight leg raise test coupled with lumbar MRI presenting no sign of disc herniation or stenosis should be per formed ultrasonography or hip MRI. Ultrasonography is useful in detecting superficial lesions. Schwannoma usually is detected as a hypoechoic solid and well-centered mass being located eccentrically on the affected nerve with preservation of its fibrillary aspect ${ }^{19}$. MRI is the gold standard diagnostic tool for peripheral nerve sheath tumors. The mass is well circumscribed and shows an isointense signal on $\mathrm{Tl}$-weighted image, with a peripheral hyperintensity and a homogeneous hypointense center on T2-weighted images. This characteristic MRI finding called a "target sign" could differentiate the schwannoma with neurofibroma, which is usually heterogenous on both T1- and T2-weighted images ${ }^{23}$. Cystic degeneration may be noted, if the tumor is subjected to pressure of injury and it can be appeared heterogeneous following gadolinium enhancement whereas schwannoma is usually enhanced homogeneously ${ }^{26}$.

The physical examination may reveal the palpable lump along the course of the sciatic nerve, which may have tenderness with a positive Tinel's sign. The neurologic examination offen demonstrates the absence of motor and sensory deficit unless the mass is very large. This is due to the lack of an infiltrative nature of the tumor and it also explains the frequent normal findings of EMG examination $^{26}$. Neural tumors associated with neurologic deficit should always raise the possibility of malignancy?.

To expose the sciatic nerve at the sciatic notch or the gluteal region, a transgluteal or a sbugluteal approach is usually employed. With the subgluteal approach, prolonged discomfort from retraction of the soft tissue and the gluteal muscle may ensue. The transgluteal approach could provide a wider surgical corridor up to the sciatic notch however, great care should be taken to muscle arteries, which if not adequately cauterized may retract within the pelvis leading to profound bleeding ${ }^{24}$.

Surgical excision is the treatment of choice for peripheral nerve schwannomas. Usually, nerve fascicles stretch over the tumor capsule and extracapsular microscopic resection along with gentle dissection of fascicles from the tumor capsule would allow complete enucleation with preservation of the nerve continuity. All intact fascicles should be identified by using intraoperative stimulation which could dete-

Table 1. Summary of previous publications describing sciatic nerve schwannoma in last 10 years

\begin{tabular}{|c|c|c|c|c|c|c|c|}
\hline Author/ & Age & $\begin{array}{l}\text { mor } \\
\text { e }\end{array}$ & $\begin{array}{l}\text { Site of the } \\
\text { Schwannoma }\end{array}$ & & Type of surgery & lesult of treatment & Follow up \\
\hline $2012^{4}$ & ars & $\mathrm{cm}$ & ximal thigh & $\mathrm{Nl}$ & Complete removal & Complete recovery & 24 months \\
\hline it et al. 201 & e/60 years & & oximal thigh & $\mathrm{Nl}$ & omplete removal & Complete recovery & 3 weeks \\
\hline & & & & 36 months & Complete removal & Complete recovery & $\mathrm{Nl}$ \\
\hline & 40 years & $\mathrm{cm}$ & & $x^{2}$ & Complete re & ete recovery & $\mathrm{NI}$ \\
\hline al. $2014^{5}$ & Female/40 years & $\mathrm{cm}$ & Middle th & onths & Complete removal & ete recovery & $\mathrm{Nl}$ \\
\hline 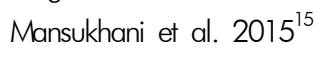 & Female/46 years & $2.5 \mathrm{~cm}$ & Proximal thigh & 48 months & Complete removal & Complete recovery & 36 \\
\hline & /40 years & $9 \mathrm{~cm}$ & & & & & $\mathrm{Nl}$ \\
\hline 16 & 27 years & & & & Cor & Co & $\mathrm{Nl}$ \\
\hline $17^{17}$ & 169 years & & al thigh & & Complete removal & te recovery & $1 \mathrm{n}$ \\
\hline & Male & & thigh & & Complete removal & te recovery & 6 months \\
\hline & 30 years & & & & & & $\mathrm{Nl}$ \\
\hline & 50 years & & & & val & te recovery & 16 months \\
\hline $2020^{24}$ & ale/47 years & & nal thign & 12 months & lete removal & Co & 24 months \\
\hline al. $2020^{24}$ & Female/79 years & $7 \mathrm{~cm}$ & Distal thigh & 6 months & Complete removal & Complete recovery & 10 months \\
\hline $2020^{27}$ & Male/27 years & $2 \mathrm{~cm}$ & Proximal thigh & 24 months & Complete removal & Complete recovery & $\mathrm{NI}$ \\
\hline Current report & Female/63 years & $3.7 \mathrm{~cm}$ & Proximal thigh & 60 months & Complete removal & Complete recovery & 3 months \\
\hline
\end{tabular}

NI; Not Indicated 
rmine the fascicles that are not functioning, and their transection does not usually end up with additional deficits. The nerve sheath is opened after a wide exposure and isolated from all adjacent neurovascular structures. The entering and exiting fascicles are cautiously identified and divided and the tumor is resected. In large tumors, it firstly necessitates an intratumor debulking and whenever functional fascicles are not possible to dissect from the capsule, it could be a better move to leave a residual mass than provoke neurologic deficit $^{14}$. However, it was reported that the majority of peripheral nerve schwannomas could be removed totally without significant neurologic deficits ${ }^{13}$.

Schwannomas are composed of two histological patterns: Antoni $A$ and Antoni B. Antoni $A$ is organized with highly vascular hypercellular areas, which are packed with Verocay bodies. It represents a circular coalescence of elongated nuclei elongated, spindle-shaped cellular nucleus. Antoni $B$ is the area where the cell mass is coarse with loose myxoid stroma ${ }^{13}$.

It is known that schwannomas have a good prognosis and a low incidence of recurrence even when the excision was incomplete ${ }^{24}$. Malignant transformation is uncommon, the risk being approximated at $1-5 \%$ in schwannomas ${ }^{3}$.

\section{CONCLUSION}

Although sciatic nerve schwannoma presents a rare occurrence, it is imperative to take into consideration whenever a patient has long-standing sciatic pain without positive findings of lumbar pathologies. MRI imaging of the nerve is mandatory for diagnosing the lesion. It is essential to outline the course of the sciatic nerve and to delineate the boundary of the mass to preserve the nerve fascicles. A standard microsurgical excision under the guidance of neurophysiologic monitoring will bring a successful removal of the tumor and good postoperative results for the patients.

Conflicts of Interest:

No potential conflict of interest relevant to this article was reported.

\section{REFERENCES}

1. Chagou A, Benameur H, Hassoun J, Jaafar A: Schwannoma of the sciatic nerve: A case report. Pan Afr Med J 37:233, 2020

2. Chahbouni M, Eloukili I, Berrady MA, Lamrani MO, Kharmaz $\mathrm{M}$, Ismail $\mathrm{F}$, et al.: Benign schwannoma of the sciatic nerve, report of 2 cases. Pan Afr Med J 18:252, 2014

3. Chikkanna JK, Gopal S, Sampath D: Mystery of Sciatica Resolved - A Rare Case Report. J Clin Diagn Res 10: Rd04-05, 2016

4. El Mekkaoui MJ, Najib A, Kharmaz M, Mahfoud M, Berrada MS, El Yaacoubi M: Schwannoma of the sciatic nerve: A rare cause of non-discal sciatica. Rev Neurol (Paris) 168:463-464, 2012

5. Eroglu U, Bozkurt M, Ozates O, Akturk S, Tuna H: Sciatic nerve schwannoma: Case report. Turk Neurosurg 24:120-122, 2014
6. Feinberg J, Sethi S: Sciatic neuropathy: Case report and discussion of the literature on postoperative sciatic neuropathy and sciatic nerve tumors. Hss J 2:181-187, 2006

7. Filler AG, Haynes J, Jordan SE, Prager J, Villablanca JP, Farahani $\mathrm{K}$, et al.: Sciatica of nondisc origin and piriformis syndrome: Diagnosis by magnetic resonance neurography and interventional magnetic resonance imaging with outcome study of resulting treatment. J Neurosurg Spine 2:99-115, 2005

8. Godkin O, Ellanti P, O'Toole G: Large schwannoma of the sciatic nerve. BMJ Case Rep. Vol 2016. 2016/11/04 ed2016

9. Gosk J, Gutkowska O, Urban M, Wnukiewicz W, Reichert P, Ziółkowski P: Results of surgical treatment of schwannomas arising from extremities. Biomed Res Int 2015:547926, 2015

10. Guedes F, Brown RS, Lourenço Torrão-Júnior FJ, Siquara-deSousa AC, Pires Amorim RM: Nondiscogenic Sciatica: What Clinical Examination and Imaging Can Tell Us? World Neurosurg 134: e1053-e1061, 2020

11. Haspolat Y, Ozkan FU, Turkmen I, Kemah B, Turhan Y, Sarar S, et al.: Sciatica due to Schwannoma at the Sciatic Notch. Case Rep Orthop 2013:510901, 2013

12. Konstantinou K, Dunn KM: Sciatica: Review of epidemiological studies and prevalence estimates. Spine (Phila Pa 1976) 33:2464-2472, 2008

13. Levi AD, Ross AL, Cuartas E, Qadir R, Temple HT: The surgical management of symptomatic peripheral nerve sheath tumors. Neurosurg 66:833-840, 2010

14. Maes R, Ledoux P, de Brouckere G: A rare cause of sciatica: Sciatic nerve schwannoma - Report of one case with long subclinical course and misleading presentation. SICOT J 6:16, 2020

15. Mansukhani SA, Butala RR, Shetty SH, Khedekar RG: Sciatic nerve schwannoma: A case report. J Orthop Surg (Hong Kong) 23:259-261, 2015

16. Mezian K, Zahora R, Vacek J, Kozak J, Navratil L: Sciatic Nerve Schwannoma in the Gluteal Region Mimicking Sciatica. Am J Phys Med Rehabil 96:e139-e140, 2017

17. Munakomi S, Shrestha P: Case Report: Sciatic nerve schwannoma - A rare cause of sciatica. F1000Res 6:267, 2017

18. Naik H, Velho V: Sciatic nerve schwannoma: A rare case. Neurol India 67:S151-s153, 2019

19. Omezzine SJ, Zaara B, Ben Ali M, Abid F, Sassi N, Hamza HA: A rare cause of non discal sciatica: Schwannoma of the sciatic nerve. Orthop Traumatol Surg Res 95:543-546, 2009

20. Pilavaki M, Chourmouzi D, Kiziridou A, Skordalaki A, Zarampoukas T, Drevelengas A: Imaging of peripheral nerve sheath tumors with pathologic correlation: Pictorial review. Eur J Radiol 52: 229-239, 2004

21. Rhanim A, El Zanati R, Mahfoud M, Berrada MS, El Yaacoubi M: A rare cause of chronic sciatic pain: Schwannoma of the sciatic nerve. J Clin Orthop Trauma 4:89-92, 2013

22. Sasaki H, Nagano S, Yokouchi M, Setoguchi T, Shimada H, Yamamoto T, et al.: Utility of intraoperative monitoring with motor-evoked potential during the surgical enucleation of peripheral nerve schwannoma. Oncol Lett 15:9327-9332, 2018

23. Suh JS, Abenoza P, Galloway HR, Everson LI, Griffiths HJ: Peripheral (extracranial) nerve tumors: Correlation of MR imaging and histologic findings. Radiology 183:341-346, 1992 
24. Telera S, Raus L, Vietti V, Pace A, Villani V, Galié E, et al.: Schwannomas of the sciatic nerve: A rare and neglected diagnosis. A review of the literature with two illustrative cases. Clin Neurol Neurosurg 195:105889, 2020

25. Thomas JE, Piepgras DG, Scheithauer B, Onofrio BM, Shives TC: Neurogenic tumors of the sciatic nerve. A clinicopathologic study of 35 cases. Mayo Clin Proc 58:640-647, 1983
26. Wolock BS, Baugher WH, McCarthy EJ: Neurilemoma of the sciatic nerve mimicking tarsal tunnel syndrome. Report of a case. J Bone Joint Surg Am 71:932-934, 1989

27. Wu WT, Chang KV, Hsu YC, Yang YC, Hsu PC: Ultrasound Imaging for a Rare Cause of Sciatica: A Schwannoma of the Sciatic Nerve. Cureus 12:e8214, 2020 